\title{
Europäisierung “Top-Down” und "Bottom-Up” und ihr Einfluss auf Minderheiten im komparativen Blickwinkel
}

\author{
Katharina Crepaz ${ }^{1, *}$ \\ 1 Max Planck Institut für Sozialrecht und Sozialpolitik \& Technische Universität München, Deutschland \\ * crepaz@mpisoc.mpg.de
}

\section{Zusammenfassung}

Der Beitrag beschäftigt sich mit dem zweidimensionalen Charakter von Europäisierungsprozessen („top-down“ und „bottom-up") und dem Ablauf dieser Prozesse sowie ihrem Einfluss auf Minderheiten in ,alten' und ,neuen" Mitgliedstaaten (deutschsprachige Minderheit in Südtirol, Italien; Bretonen in Frankreich; deutsche Minderheit in Schlesien, Polen und italienische Minderheit in Istrien, Kroatien). Dazu werden strukturierte Interviews mit MinderheitenvertreterInnen und EU-BeamtInnen sowie parlamentarische Fragen an die EU-Kommission mittels Process Tracing analysiert. Während sich die grundsätzlichen Annahmen über eine Vorherrschaft von „Top-Down“-Prozessen in „neuen“ und „Bottom-Up“-Prozessen in „alten" Mitgliedstaaten bewahrheitet haben, sind die Erkenntnisse über die Präsenz von „Top-Down“-Prozessen in „alten“ Staaten (Fallbeispiel Südtirol) sowie das Vorhandensein einer starken Zivilgesellschaft in „neuen“ Staaten (Schlesien, Istrien) durchaus überraschend.

\section{Schlüsselwörter}

Europäisierung, Minderheiten, Zivilgesellschaft, "bottom-up" Prozesse

\section{Europeanization "top-down" and "bottom-up" and its influence on minorities - a comparative perspective}

\begin{abstract}
The present paper looks at Europeanization as a two-dimensional process, encompassing both "top-down" and "bottom-up" processes, how these developments take place, as well as their impact on minorities in 'old' and' new' member-states (the German-speaking minority in South Tyrol, Italy; the Bretons in France; the German minority in Silesia, Poland; the Italian minority in Istria, Croatia). Structured interviews with minority representatives and EU officials as well as parliamentary questions for answer from the European Commission will be analyzed with process tracing methodology. While the hypotheses regarding a prevalence of "top-down" processes in 'new' and of "bottom-up" processes in 'old' member-states are generally confirmed, the presence of "top-down" processes in 'old' member-states (South Tyrol) and the involvement of a strong civil society in "new" member-states also provide unexpected results.
\end{abstract}

\section{Keywords}

Europeanization, minorities, civil society, "bottom-up" processes

The author has declared that no competing interests exist. 


\section{Einleitung}

"Einheit in Vielfalt" gilt als eines der Grundprinzipien der EU - es beinhaltet den Anspruch, das sehr heterogene kulturelle Erbe des Kontinents zu bewahren, aber auch gemeinsame, verbindende Elemente zu finden und hervorzuheben. Eine Zielgruppe dieses ambitionierten Vorhabens sind die nationalen und autochthonen Minderheiten, die in den verschiedenen Mitgliedstaaten leben. Laut FUEN (Föderalistische Union Europäischer Nationalitäten) gibt es in Europa 300 Minderheiten, und jede/r siebte Europäer/in ist Teil einer Minderheit oder spricht eine Minderheitensprache. Zu den 23 offiziellen Amtssprachen der EU kommen 60 Regional- oder Minderheitensprachen hinzu; von den über 90 in Europa gesprochenen Sprachen sind 57 Nationalsprachen, während 53 als "staatenlose" Sprachen gelten, d.h. sie sind in keinem Staat offizielle Amtssprache. Das Beispiel der Sprachen zeigt bereits, wie unterschiedlich die Ausgangspositionen europäischer Minderheiten sind; so verschieden wie Katalanisch mit seinen 6 Millionen SprecherInnen und Ladinisch mit knapp 30.000 sind auch die politischen Präferenzen der Mitgliedstaaten in der Minderheitenpolitik, die implementierten Schutzstandards, die Relevanz der Minderheit auf dem nationalen Parkett, die Möglichkeiten zur politischen Partizipation usw. Diese Vielfalt unter einem gemeinsamen Ansatz zu versammeln ist angesichts sehr unterschiedlicher Positionen ein herausforderndes Unterfangen; als einer der Referenzpunkte dienen seit 1993 die Kopenhagen Kriterien, die unter anderem „die Wahrung der Menschenrechte sowie die Achtung und den Schutz von Minderheiten" als Beitrittsvoraussetzungen für neue Mitgliedstaaten festlegen.

Die Mittel- und Osteuropäischen Staaten der „BigBang“-Erweiterung 2004 konstituierten die ersten Fälle, bei denen die EU im Beitrittsprozess spezifisch auf die Wahrung von Mindeststandards für Minderheitenschutz achtete. In der Wissenschaft bestand auf diesem Gebiet fortan die Dichotomie zwischen prä- und post-2004 Mitgliedstaaten bzw. Ländern, deren Minderheitenpolitik keiner Evaluation unterzogen wurde und jenen, bei denen politische Konditionalität als Druckmittel verwendet wurde, oder zumindest als solches zur Verfügung stand. Maßnahmen zum Minderheitenschutz bleiben, sofern sie sich über die Dimension der Nicht-Diskriminierung hinaus erstrecken, weiterhin ein rein politisches und kein rechtliches Beitrittskriterium - ein Mangel an Kompetenzen seitens der EU, der ein wirksames „Einmischen" nach dem Beitrittsprozess quasi unmöglich macht.

Eine Überwindung der in der Literatur vorherrschenden Dichotomie zwischen "alten“ und „neuen“ Mitgliedstaaten und ihrer jeweiligen Minderheitenpolitik ist der erste Anspruch, den dieser Beitrag erfüllen möchte; es soll gezeigt werden, dass gerade ein Brücken-bildender komparativer Ansatz neue Erkenntnisse zu unterschiedlichen und ähnlichen Entwicklungen liefern kann. Das zweite Forschungsziel ist ein differenzierterer Blick auf Europäisierungsprozesse, welche häufig nur als Einflussnahme der EU auf die nationale und subnationale Ebene gesehen werden, und deren Analysefokus daher auf Bereiche innerhalb des acquis communautaire beschränkt bleibt. Es soll gezeigt werden, dass es Europäisierungsprozesse auch außerhalb des Rechtsbestandes gibt, bzw. dass acquis-Normen durch Spill-Over-Effekte auch Politikfelder beeinflussen, in denen die EU keine Kompetenz hat. Auch Europäisierung „von unten“ und Vernetzung der Zivilgesellschaft wurden bisher hauptsächlich für Themen innerhalb des acquis untersucht, sie finden allerdings ebenfalls außerhalb statt. Europäisierung soll im Rahmen dieses Beitrags als zweidimensionaler Prozess gesehen werden, welcher sowohl „Top-Down“ (von der EU-Ebene auf die nationale und subnationale Ebene) als auch „Bottom-Up“ (subnationale oder zivilgesellschaftliche Akteure nutzen die EU-Ebene für die Verfolgung ihrer Ziele) europäische Minderheiten in "alten" und "neuen" Mitgliedstaaten beeinflusst und direkten und indirekten Einfluss auf ihre Lebenssituation hat. Um die Thematik im komparativen Blickwinkel zu beleuchten, werden nach einem most dissimilar cases-Design vier sehr unterschiedliche europäische Minderheiten miteinander verglichen, zwei in "alten" (die deutschsprachige Minderheit in Südtirol, Italien und die Bretonen in Frankreich) und zwei in "neuen" (die deutsche Minderheiten in Schlesien, Polen und die italienische Minderheit in Istrien, Kroatien) Mitgliedstaaten.

\section{Europäisierung als zweidimensionaler Prozess}

Das klassische Verständnis von Europäisierung bezeichnet meist den Einfluss politischer Prozesse bzw. Normen auf EU-Ebene auf die nationale und subnationale Ebene; der Grad an fit oder misfit ist dabei relevant für die Möglichkeiten zur Adaptierung und Implementierung (Börzel und Risse 2003). Eine häufig zitierte Definition von Graziano und Vink (2007, 7) spricht von „domestic adaptation to European regional integration", Schimmelfennig und Sedelmeier $(2004,7)$ sehen Europäisierung als „a process in which states adopt EU-rules“. Diesen Definitionen gemein ist ein sehr „Top-Down“ geprägtes Verständnis von Europäisierungsprozessen, bei dem es immer um die Einflussnahme der europäischen auf andere Ebenen geht. Cowles, Caporaso und Risse (200I, 4) wählen einen umfassenderen Begriff:

[...] the emergence and development at the European level of distinct structures of governance, 
that is, of political, legal and social institutions associated with political problem solving that formalize interactions among the actors, and of policy networks specializing in the creation of authoritative European rules

- es geht nicht mehr nur um die Art des Einflusses, sondern um die Entstehung neuer Strukturen im Mehrebenensystem. Zudem betonen sie bereits den zweidimensionalen Charakter von Europäisierung: "the causal processes go both ways - activities at the European level affect the domestic level and vice versa."

Einen noch breiteren Ansatz verfolgen Radaelli und Exadaktylos (2OIO, 193): Europäisierung inkludiert hier auch

shared beliefs and norms which are first consolidated in the EU policy process and then incorporated into the logic of domestic (national and subnational) discourse, identities, political structures and public policies. Thus, Europeanization is an interactive process rather than a simple process of unidirectional ,reaction" to Europe. It covers both the notion of Europeanization as domestic impact of Europe (or pressure) and Europeanization as creative usages of Europe.

Der Europäisierungsbegriff wird also auf ein zweidimensionales Konzept erweitert, in dem nicht nur Einflussnahme von der EU-Ebene auf die nationale und subnationale Ebene analysiert wird, sondern auch die "Nutzung" der europäischen Arena durch andere Akteure eine Rolle spielt. Della Porta und Caiani sehen „Bottom-Up" Prozesse als Europäisierung "of and by civil society" (2009, 25); Europa gilt in diesem Fall konstruktivistisch als sich bildende Polity, in welcher das Thema Legitimität durch die Einbindung sozialer Bewegungen und zivilgesellschaftlicher Organisationen behandelt werden kann. Durch „Europeanization by externalization" können Themen, die von Akteuren auf die europäische Ebene "gehoben“ wurden, dazu genutzt werden, Druck auf nationale Entscheidungsträger in verschiedenen Mitgliedstaaten auszuüben. McCauley definiert Europäisierung „von unten“ als „re-orientation of a (sub) national actor's champ d'activité towards supranational institutions, politics, and/or policy-making" (2OII, IO20). Solche Prozesse der Orientierung lassen sich vornehmlich in Politikfeldern beobachten, in denen die EU Entscheidungskompetenzen hat (z.B. transnationale zivilgesellschaftliche Bewegungen zum Umweltschutz, oder gegen GMO-Lebensmittel).

Eine Zusammenarbeit und Vernetzung auf europäischer Ebene ist aber auch in Bereichen außerhalb des acquis communautaire möglich, z.B. durch den Austausch von Best Practices, oder die Nutzung der EU als Forum für Bewusstseinsbildung zu bestimmten Problemstellungen. In einem solchen Kontext können auch "transnational advocacy networks" enstehen (Keck/Sikkink 1998), die internationale Zusammenarbeit zum Erreichen eines gemeinsamen Ziels beinhalten. Zudem gibt es europäische Normen, die als rechtlicher Rahmen von Einfluss sein können, auch wenn sie nicht direkt ein bestimmtes Politikfeld betreffen - so hat die EU z.B. keine Kompetenz im Bereich Minderheitenschutz, sehr wohl aber Normen zur Anti-Diskriminierung, die Minderheiten und ihre tägliche Lebensrealität direkt ansprechen und beeinflussen. Der Europäisierungsbegriff und die Europäisierungsforschung bewegen sich also weg vom klassischen Verständnis der Einflussnahme Europas auf die Mitgliedstaaten hin zu einem breiteren, prozessorientierten und zweidimensionalen Ansatz, in dem auch non-acquis Politikfelder und nationale sowie subnationale Akteure Platz finden.

Ein weiter gefasster Begriff von Europäisierung, ihren Erscheinungsformen und möglichen Konsequenzen repräsentiert auch die theoretische Ausgangsposition dieses Papers. Minderheiten werden in ihrem Handlungsspielraum, aber auch in ihrer politischen Ausrichtung und den Rahmenbedingungen ihres Wirkens stark von der europäischen Ebene beeinflusst; im Gegenzug nutzen sie diese allerdings auch zur Vernetzung und zum gemeinsamen Lobbying, insbesondere wenn der Nationalstaat als nicht kooperativ angesehen wird bzw. Anliegen von mehreren, vielleicht auch größeren oder politisch relevanteren Minderheiten getragen werden. Wie die Interaktion und die "Nutzung" Europas durch Minderheiten konkret aussehen, ist von Fall zu Fall sehr verschieden und nicht zuletzt auf nationalstaatlicher Ebene anzusiedelnden scope conditions abhängig. Im folgenden Theorieteil sollen daher einige, bewusst breit formulierte Hypothesen aufgestellt werden, wie sich $\mathrm{Eu}$ ropäisierungsprozesse auf Minderheiten in „alten“ und „neuen“ Mitgliedstaaten auswirken könnten.

\section{3. „Alte“ und "neue“ Mitgliedstaaten im komparativen Blickwinkel}

Anspruch dieses Artikels ist es, einen zweidimensionalen Europäisierungsbegriff zu verfolgen, der sowohl „Top-Down“- als auch „Bottom-Up“-Entwicklungen einschließt, und die in der Literatur vorherrschende Dichotomie zwischen „alten“ und "neuen“ Mitgliedstaaten hinsichtlich Minderheitenpolitik durch einen vergleichenden Fokus zu überwinden. Zudem soll dargestellt werden, dass Europäisierungsprozesse auch in Politikfeldern außerhalb des acquis präsent sind. Im Forschungsinteresse stehen also der Einfluss von zweidimensionalen Europäisierungsprozessen auf sehr verschiedene Minderheiten sowie mögliche Faktoren auf 
nationaler Ebene, die als Veto-Player oder Katalysatoren für solche Prozesse fungieren können.

Im europäischen Mehrebenensystem kann sich Europäisierung auf verschiedenen Leveln abspielen bzw. von verschiedenen Akteuren durchgeführt werden. Im klassischen Europäisierungsbegriff wurde der Fokus auf die Einflussnahme von „oben nach unten“ gelegt; daher sollen hier gerade der partizipative Aspekt und Prozesse von "unten nach oben“ besondere Aufmerksamkeit erhalten. Die zu untersuchenden Minderheiten unterscheiden sich stark in Art und Vorhandensein von Minderheitenschutz, Größe, politischer Relevanz, Autonomie- oder Selbstverwaltungsmöglichkeit, Ressourcen usw.: Während in Südtirol ein gut ausgebautes Modell territorialer Autonomie vorherrscht, gibt es in Schlesien kulturelle Autonomiekonzepte und in Istrien ein Prozent-Modell. In der Bretagne verhindert die republikanische französische Auffassung der Nation regionale Selbstverwaltungsaspekte. Das Spektrum der zu behandelnden Minderheiten wurde bewusst so konzipiert, von sehr weitreichenden Schutzmechanismen bis zur Absenz positiver Maßnahmen sehr unterschiedliche Fälle einzuschließen und gerade durch diese Unterschiede einen spannenden und ambitionierten Vergleich liefern zu können. Das Forschungsdesign folgt also einem most dissimilar cases Ansatz, bei dem möglichst unterschiedliche Fallstudien hinsichtlich der Erklärungskraft einer Variable, in diesem Fall Europäisierungsprozesse, untersucht werden.

Als Daten dienen vornehmlich qualitative strukturierte Interviews mit MinderheitenvertreterInnen ( 5 Interviews pro Minderheit), die von Januar bis Juli $2014 \mathrm{im}$ Rahmen meines Dissertationsprojektes (Crepaz 2016) geführt wurden. Es handelte sich bei den InterviewpartnerInnen um politische RepräsentantInnen der Minderheiten auf lokaler, regionaler und europäischer Ebene, sowie um führende Mitglieder von Minderheiten-NGOs (z.B. der Föderalistischen Union Europäischer Nationalitäten, dem Kuzhul Ar Brezhoneg, sowie dem Verband der deutschen sozial-kulturellen Gesellschaften in Polen). Die InterviewpartnerInnen sind also RepräsentantInnen kollektiver Akteure, die für sich beanspruchen, zumindest bis zu einem gewissen Grad für die Belange der gesamten Minderheit sprechen zu können. Da sich die Fragen auf politische Entwicklungen in den jeweiligen Ländern beziehen, kommt auch die persönliche Meinung der Befragten in den Interviews zu tragen. Zur Triangulierung und um möglichen Bias zu vermeiden, wurde zudem eine Analyse parlamentarischer Fragen von Minderheiten-MEPs an die EU-Kommission durchgeführt, sowie Interviews mit BeamtInnen der EU-Kommission geführt (DG Justiz und DG Erweiterung, jeweils zwei InterviewpartnerInnen), und die dadurch erhobenen Daten mit den Interviewdaten der MinderheitenvertreterInnen verglichen. Durch eine zusätzliche Analyse der zeitlichen Entwicklung soll zudem festgestellt werden, in welchen Zeiträumen der EU-Einfluss am größten war und wie sich die Möglichkeiten zur Zusammenarbeit und Vernetzung auf der Zeit-Achse entwickelt haben. Mit insgesamt 24 Interviews kann die Studie als kleinere qualitative Analyse eingestuft werden. In Kombination mit der Auswertung der parlamentarischen Fragen und durch die Triangularisierung sowie den ExpertInnenstatus der Befragten wurde der Sättigungspunkt dennoch erreicht, da die jeweils wichtigsten kollektiven Akteure im Europäisierungsprozess abgedeckt wurden.

Anhand der bereits bestehenden Literatur zum Thema sowie der Ausgangsposition vor Durchführung der Interviews lassen sich folgende Hypothesen bilden:

I. „Bottom-up“-Prozesse treten häufiger in den „alten“ Mitgliedstaaten auf. Da die EU hier im Beitrittsprozess nicht eingegriffen hat bzw. noch nicht über Konditionalität als Werkzeug verfügte, ist größerer Einfluss der europäischen Ebene unwahrscheinlich. Da das Thema zudem stark relevant für die nationale Identität ist, könnte ein ablehnender Nationalstaat eine Gate-Keeper-Funktion einnehmen und versuchen, unerwünschte Prozesse vor dem „Import“ auf die nationale Ebene zu stoppen. Europäisierungsprozesse geschehen hauptsächlich durch eine starke Zivilgesellschaft, die es schafft, Themen in die europäische Arena zu tragen.

2. Ist die Kommunikation zwischen der Minderheit und dem Nationalstaat gestört oder ist er nicht bereit, auf die Wünsche der Minderheit einzugehen, ist ein Anstieg von Europäisierungsbemühungen zu erwarten. Minderheiten könnten das Thema auf die europäische Ebene bringen, um von dort aus Druck auf den Mitgliedstaat auszuüben („Europeanization by externalization“). Gibt es hingegen eine gute Kommunikationsbasis zwischen Minderheit und staatlicher Ebene, können viele Probleme bereits innerstaatlich gelöst werden und eine Diskussion auf europäischem Niveau wird hinfällig.

3. Für die "neuen“ Mitgliedstaaten wird eine Prävalenz von „Top-Down“-Prozessen angenommen; hier konnte die EU durch Konditionalität im Beitrittsprozess direkt Einfluss auf die Minderheitenpolitik nehmen. Gegen solche von „oben“ installierten Normen könnte es nach dem Beitritt allerdings einen Backlash geben. Die bisherige Forschung (z.B. Schimmelfennig 2008) konstatiert zudem eine Schwäche der Zivilgesellschaft in den mittel- und osteuropäischen Ländern, die es schwieriger macht, Themen auf die europäische Agenda zu setzen.

4. Einflussfaktoren auf nationalstaatlicher Ebene können als Gate-Keeper oder Katalysatoren agieren. Die Haltung des jeweiligen Nationalstaates gegenüber positiven Maßnahmen zum Schutz von Minder- 
heiten sowie die Verwurzelung von Vielfalt in der nationalen Identität sind dabei wichtige Konzepte, können Europäisierungsprozesse aber nicht allein auslösen oder sie stoppen.

Zur Analyse und Auswertung der durch Interviews, parlamentarische Anfragen und offizielle Dokumente gesammelten Daten soll Process Tracing herangezogen werden. Process Tracing ist besonders für qualitative Forschungsansätze geeignet, da es ermöglicht, sehr genaue Aussagen über detaillierte Fallstudien zu machen (Beach/Pedersen 20I3). Mechanismen und ihre Bestandteile sowie die einzelnen Schritte sich abwickelnder Prozesse können analysiert und hinsichtlich Kausalität der Zusammenhänge evaluiert werden; die Identifizierung von besonders wichtigen Ereignissen, Kontexten oder Turning Points wird möglich. Eine reine Cause-Effect Betrachtung, wie sie z.B. die Ko-Variationsmethode bietet, trägt der Komplexität des Themas und der Orientierung an Europäisierung als Prozess nicht ausreichend Rechnung. Eine umfassende Process Tracing Analyse aller Aspekte der Entwicklung der jeweiligen Minderheitensituation liegt dem Forschungsdesign der größeren Studie zu Grunde (Crepaz 2016), kann im Rahmen dieses Artikels aber nicht abgebildet werden; vielmehr soll auf jüngere Entwicklungen und spezifische Auswirkungen und Ausprägungen von Europäisierungsprozessen eingegangen werden.

Zunächst folgen nun eine genauere Vorstellung der Fallstudien mit zum Verständnis notwendigen Hintergrundinformationen sowie ein Blick auf die Hypothesen im Kontext der jeweiligen Minderheiten. Abschließend werden die durch die Auswertung gewonnenen Erkenntnisse in komparativen Fokus gesetzt und die verschiedenen Mechanismen bzw. Prozesse miteinander verglichen.

\section{Fallstudien}

\section{a) Südtirol}

Die deutschsprachige Minderheit in Südtirol kann, zusammen mit der italienischen Minderheit in Istrien, als die am weitgehendsten und besten geschützte Minderheit im Rahmen der Fallstudien angesehen werden. In Italien ist der Schutz der sogenannten "sprachlichen Minderheiten" in Artikel 6 der Verfassung verankert; zudem gibt das Gesetz 482/1999 an, welche Gruppen zu den „historischen sprachlichen Minderheiten“ zählen und wo diese leben - es werden ausschließlich territorial konzentrierte Volksgruppen geschützt, daher finden z.B. die in Italien auch als autochthone Minderheit vorkommenden Roma hier keine Erwähnung. Die meisten Minderheiten leben in Regionen mit Sonderstatut (z.B. Aostatal, Friaul-Julisch-Venetien, Trentino-Südtirol), welche im Vergleich zu Regionen mit Normalstatut mehr Kompetenzen aufweisen. Für Minderheiten ist die Einbindung in eine Region mit Sonderstatut aufgrund der größeren Kompetenzen auf lokaler Ebene von Vorteil; ein gutes Beispiel dazu sind die Ladiner - während die im Trentino und in Südtirol ansässigen Gruppen gut geschützt sind und z.B. im Primarbereich muttersprachliche Bildung erhalten, sind die Möglichkeiten zum Spracherwerb und zur Sprachpflege im angrenzenden Belluno (in der Region Veneto) wesentlich schlechter, was sich bereits in Sprachverlust niederschlägt.

Die Schutzmaßnahmen für Südtirol gehen über den generellen gesetzlichen Rahmen hinaus, da die deutschen und ladinischen Minderheiten zusätzlich durch den Pariser Vertrag von 1946 international abgesichert sind. Weitere internationale Aufmerksamkeit erfuhr die Südtirol-Thematik in den I96oer Jahren, als der österreichische Außenminister Bruno Kreisky die Angelegenheit vor dem Hintergrund nicht eingehaltener Bestimmungen des ersten Autonomiestatuts sowie des aufkeimenden politischen Terrorismus vor die UNVollversammlung brachte. Durch die Ausarbeitung des Zweiten Autonomiestatuts (seit 1972 in Kraft), welches weitreichende Kompetenzen auf Provinz- statt auf Regionalebene vorsah, wurde die Gewaltperiode der 6oer Jahre überwunden und die politische Ausrichtung ging in Richtung Autonomie anstatt Rückkehr zu Österreich. Neben der internationalen Verankerung der Schutzmaßnahmen weist Südtirol eine weitere Besonderheit auf, nämlich dass die Minderheit innerhalb der Provinz eine Mehrheitsstellung einnimmt: Laut Volkszählung 2012, in der auch zur Berechnung des Proporzsystems für die Vergabe der öffentlichen Stellen die Sprachgruppenzugehörigkeit anonym erhoben wird, erklärten sich $69,41 \%$ als deutschsprachig, $26,06 \%$ als italienischsprachig und 4,53\% als ladinischsprachig (ASTAT 20I3). Diese Sonderstellung und die Erlangung primärer Gesetzgebungskompetenz in vielen Bereichen (Tourismus, Gesundheit, Kindergärten, Berufsbildung etc.) legten den Grundstein für eine ausgebaute Autonomie, welche jedoch durch die Austeritätsmaßnahmen im Rahmen der Finanzkrise zunehmend beschnitten wird. In Anbetracht dieser Entwicklungen lässt sich eine „Territorialisierung" (Pallaver 20I4) der Autonomie feststellen: Wurde früher von den politischen Eliten der Fokus auf den ethnisch unterschiedlichen Charakter des Gebiets gelegt, so sollen jetzt nicht mehr nur die Angehörigen der Minderheit, sondern alle auf dem Gebiet lebenden Menschen angesprochen werden. Der ethnische Charakter der Autonomie weicht also einem territorialen Gedanken, der alle Bevölkerungsgruppen einschließt, auch in den politischen Diskursen der Minderheitenvertreter gegenüber der Regierung in Rom. Pallaver weist in diesem Zusammenhang auch auf die wichtige Rolle der EU für diese Entwicklung hin: Die EU stellt den sicheren 
Rahmen für eine Vertrauensbasis zwischen den Volksgruppen dar, die Minderheit weiß, dass sie keine Repression zu befürchten hat und kann daher den Fokus auf die reine Ethnizitäts-Cleavage aufgeben (Pallaver 20I4).

\section{b) Bretagne}

Der Fall der Bretonen gestaltet sich sehr unterschiedlich zu jenem Südtirols. Obwohl es sich bei beiden Fallstudien um Minderheiten in „alten“ Mitgliedstaaten handelt, gibt es große Unterschiede: Im Gegensatz zu Italien verweist Frankreich in seiner Verfassung nicht auf Minderheitenschutz, sondern auf das einige Staatsvolk und dessen Sprache, Französisch (Artikel 2). Es wird also klar, dass im radikalen republikanischen Gleichheitsgrundsatz nur wenig Platz für die Anerkennung kultureller Diversität bleibt. Das Bretonische, eine keltische Sprache, kann nur in zusätzlichen Unterrichtsstunden bzw. privaten Institutionen erlernt werden, und ist daher stark von Sprachverlust betroffen: Die UNESCO klassifiziert Bretonisch als „stark gefährdet“. Bei den jüngeren Generationen scheint es aber einen positiven Trend zu geben; so stieg die Anzahl der SchülerInnen, die an zweisprachigen Klassen teilnahmen, zwischen 2006 und 2013 um 33\% an (Office Publique de la Langue Bretonne 2013). Der Jugend die Sprache näherzubringen ist eines der wichtigsten Ziele der Minderheit, da das Bretonische häufig mit einem niedrigen Prestige als Sprache der älteren, ländlichen Bevölkerung zu kämpfen hat. Genaue Zahlen zu den SprecherInnen gibt es nicht, auch aufgrund des Verbots ethnische Daten zu sammeln; die Schätzungen bewegen sich zwischen 300.000 und einer Million. Ähnlich wie in Südtirol gab es auch in der Bretagne politischen Terrorismus, der auf eine Abtrennung des Gebietes von Frankreich abzielte; die letzten Anschläge erfolgten hier erst relativ spät (Anfang der 2oooer Jahre). Mittlerweile hat sich eine aktive Zivilgesellschaft gebildet, die vor allem in den Protesten gegen die französische Agrarpolitik sehr präsent ist.

Als momentan wichtigstes Anliegen der Minderheit kann die Ratifizierung der Charta für Regional- und Minderheitensprachen des Europarats gesehen werden, welche Frankreich bereits 1999 unterzeichnet hat. In den Reservations, die bei der Unterzeichnung gemacht wurden, unterstreicht Frankreich, dass die Charta keine gruppenspezifischen Rechte gewährt bzw. keine Minderheiten offiziell anerkennt und somit mit der Präambel der französischen Verfassung kompatibel sei; trotz dieses Verständnisses verweigerte der Staatsrat die Verwendung des Begriffes "Gruppen“, der auf eine kollektive Dimension hinweise, und die Möglichkeit der Nutzung von Regional- und Minderheitensprachen im amtlichen Kontext. Eine Ratifizierung der Charta konnte bisher nicht durchgeführt werden, obwohl Präsident Hollande diese als eines seiner Wahlziele beworben hatte. Anfang
2014 stimmte die Nationalversammlung einem Verfassungsgesetz $\mathrm{zu}$, welches die Ratifizierung der Charta ermöglicht; der Senat lehnte diese 2015 allerdings ab. Bis heute (März 20I7) sind keine weiteren signifikanten Schritte hin zu einer Ratifizierung gemacht worden.

\section{c) Schlesien}

Die deutsche Minderheit in Polen lebt Großteils in der Gegend um Oppeln, ein kleinerer Teil auch im Raum Danzig und Masuren, und zählt heute ca. 300.00o350.00o Mitglieder. Im Gegensatz zu Frankreich dürfen in Polen ethnische Daten erhoben werden, und in der Volkszählung wird die Frage nach der ethnischen $\mathrm{Zu}$ gehörigkeit gestellt - die Beantwortung ist allerdings freiwillig und Mehrfachnennungen sind möglich, man kann sich also z.B. sowohl als deutsch als auch als schlesisch oder polnisch erklären. In der Volkszählung 20II erklärten sich 153.000 Menschen der deutschen Volksgruppe zugehörig; 847.000 erklärten sich als Schlesier, ein signifikanter Anstieg im Vergleich zu $173.000 \mathrm{im}$ Jahr 2002 (Central Statistical Office of Poland 2014). Es ist wahrscheinlich, dass viele Angehörige der deutschen Minderheit eine "neutralere“, regional ausgerichtete schlesische Zugehörigkeit bevorzugen, welche nicht mit der historischen Belastung der deutschen Identität zu kämpfen hat. Zudem ist gerade für die junge Generation eine regionale Verankerung häufig wichtiger als eine Selbst-Identifikation als Deutsche/r, welche als weiter weg von der eigenen Lebensrealität angesehen wird (Cordell/Wolff 2005, 268).

Die Minderheit ist im Verband der deutschen Gesellschaften (VdG) organisiert, der auf lokaler Ebene über 600 Deutsche Freundeskreise betreibt; diese kümmern sich um die Weitergabe von Sprache und Kultur. Schlesien hatte seit jeher einen multikulturellen Charakter und ist auch heute auf drei Staaten verteilt (Polen, Deutschland, Tschechien). Im I9. Jahrhundert kamen vermehrt Deutsche in die Region, um in der Schwerindustrie zu arbeiten; nach dem I. Weltkrieg bildete sich dadurch eine große deutsche Minderheit in dem vom Völkerbund verwalteten Gebiet, welches schließlich nach einer Volksabstimmung zwischen Deutschland und Polen aufgeteilt wurde. Mit dem deutschen Einmarsch 1939 begann die Unterdrückung der polnischen Bevölkerung, während nach Kriegsende viele Deutsche ihre Heimat verlassen mussten. Die deutsche Sprache wurde verboten; in den Interviews wurde deutlich, dass die Nachkriegsgeneration ab Erreichen des Schulalters nur noch Polnisch sprach, und deshalb auch kaum in der Lage war, das Deutsche an ihre Kinder weiterzugeben. Sprachverlust ist daher eines der Hauptprobleme der Minderheit, dem mit frühkindlicher Bildung und zweisprachigen Schulklassen entgegenzuwirken versucht wird. Bis 1990 hatten I,I Mio. Menschen deutscher Herkunft Polen verlassen; viele nahmen die Möglichkeit wahr, einen 
deutschen Pass zu erhalten und in die Bundesrepublik auszuwandern. Der Wegzug von jungen, qualifizierten Menschen ist auch heute noch ein großes Problem für die Minderheit, deren Mitgliederzahlen sich so weiter verringern (Cordell/Wolff 2005, 264-267).

Für die Vertreter der Minderheit gelten das Ende des Kommunismus und der EU-Beitritt als die beiden wichtigsten Ereignisse für die weitere Entwicklung der Situation. Nach Ende des kommunistischen Regimes konnte wieder Deutsch gelernt werden, im Rahmen von drei zusätzlichen Schulstunden. Die heutige Führungsriege kritisiert hierbei das Verhalten ihrer Vorgänger, sich mit diesem geringen Zugeständnis zufrieden zu geben, und forciert eine zweisprachige Schule mit möglichst rein muttersprachlicher frühkindlicher Bildung. Während die Minderheit immer noch mit dem negativen Image der ehemaligen Besatzer zu kämpfen hat, wird das zweisprachige Schulmodell als ausbauwürdig angesehen und auch von polnischen Familien genutzt. Auch die schlesische Autonomiebewegung sieht man innerhalb der Minderheit durchaus positiv; es wird aber aus Angst vor Separatismusvorwürfen darauf verzichtet, sich zu stark einzubringen. Im Jahr 2005 wurde nach einer Vorbereitungsphase von mehr als 17 Jahren das Gesetz zum Schutz der nationalen Minderheiten verabschiedet; laut Angehörigen der Minderheit kam dem EU-Beitritt dabei eine wichtige Rolle beim Erreichen eines Kompromisses $\mathrm{zu}$.

\section{d) Istrien}

Die italienische Minderheit in Kroatien ist relativ klein und größtenteils territorial auf Istrien konzentriert; ein kleiner Teil (I.000-4.00o Menschen) lebt auch in Dalmatien. Die Minderheit hat laut Volkszählung $201 \mathrm{I}$ I7.807 Mitglieder und stellt somit $0,42 \%$ der Gesamtbevölkerung; sie ist nach Serben (4,36\%) und Bosniern (o,73\%) die drittgrößte Minderheit des Landes. Die Anzahl der Italiener in Kroatien ist im Sinken begriffen, 200I hatten sie noch 19.636 Mitglieder - es wird interessant sein zu sehen, welche Auswirkungen der EU-Beitritt Kroatiens auf die Entwicklung der Minderheit hat. In Kroatien gibt es wie auch in Polen die Möglichkeit, eine „regionale Affiliation“ zu erklären; 2OII machten 27.225 Menschen davon Gebrauch (Croatian Bureau of Statistics 20I3, IIO); eine Stärkung regionaler Identitäten und Zugehörigkeiten ist also auch in Kroatien spürbar. In den Berichten der EU-Kommission wird häufig auf die Lage der serbischen Minderheit eingegangen, da es hier die größten Probleme hinsichtlich Diskriminierung, Unterrepräsentation im öffentlichen Dienst und auch Rückführung von Flüchtlingen gab. Ein weiteres wichtiges Thema der Reports sind die Roma; die italienische Minderheit wird kaum erwähnt, einer der für Kroatien zuständigen Beamten der DG Erweiterung erinnert sich, dass es hier auch während der Beitrittsverhandlungen kaum Diskussionspunkte gab, da die Minderheit bereits gut geschützt war.

Im Gegensatz zu anderen kommunistischen Ländern hatte Jugoslawien eine für kulturelle Diversität sehr offene Ausrichtung; Artikel I der Verfassung nannte Kroatien den "Nationalstaat der Kroaten, Serben in Kroatien und anderer Nationalitäten“. Ähnlich Schlesien verließen viele Italiener nach 1945 Kroatien; ca. 250.00o Menschen wanderten aus Istrien und 45.000 aus Dalmatien nach Italien aus (Anderini 2006, 38). Eine weitere Analogie bildet die multikulturelle Identität Istriens, wo ein Zusammenleben verschiedener Volksgruppen ebenfalls lange selbstverständlich war. Heute ist die Region über Italien, Slowenien und Kroatien aufgeteilt und arbeitet wieder verstärkt grenzüberschreitend zusammen, z.B. in der Euregio „Ohne Grenzen“. Im Unterschied zu Polen gab es allerdings keine Unterdrückung der Sprache und Kultur, die Verfassung garantierte muttersprachlichen Unterricht, proportionale Repräsentation in der lokalen Verwaltung, Verwendung der Muttersprache bei Behörden etc. Die italienische Minderheit besaß also den Großteil der heutigen Schutzmechanismen bereits in der jugoslawischen Zeit und es konnte eine gewisse Kontinuität erfolgen, die in Schlesien durch die kommunistische Repression nicht möglich war.

Als 199I Kroatien und Slowenien ihre Unabhängigkeit erklärten und daraufhin der Balkankrieg ausbrach, wurde sich auch die italienische Minderheit wieder stärker ihrer Identität bewusst; allerdings war diese bereits damals stark regional geprägt und inkludierte auch die in Istrien lebenden Kroaten und Slowenen (Anderini 2006, 39-40). Istrien war die einzige Region, in der Tudjmans HDZ keinen Wahlsieg davontragen konnte; obwohl kein primärer Kriegsschauplatz, litt das Gebiet stark unter dem Wegfall des Tourismus als wichtige Einnahmequelle. Das Boniver-Gesetz (Möglichkeit zur Auswanderung und Arbeitserlaubnis in Italien für Angehörige der Minderheit) wurde 1993 verabschiedet; von den 4.000 Menschen, die sich dafür registrierten, verließ letztendlich aber nur ein kleiner Teil das Land, hauptsächlich als Wochenendpendler (Anderini 2006, IO5-III). Seit 1992 verfügt die Minderheit über einen garantierten Vertreter im kroatischen Sabor, 1996 wurde ein bilateraler Vertrag zwischen Kroatien und Italien über die Rechte der Minderheiten unterschrieben.

Kroatiens Weg in die EU war zunächst sehr langsam, nach Tudjmans Tod und dem Regierungswechsel im Jahr 2000 konnten größere Fortschritte erzielt werden. Im Jahr 2002 wurde das Verfassungsgesetz über die Rechte nationaler Minderheiten verabschiedet. Bei den Staaten des Westbalkans wendet die EU laut Beamten der EU-Kommission (DG-Erweiterung) ein neues Verhandlungsverfahren an, in welchem die Punkte Menschenund Minderheitenrechte als erstes eröffnet und als letztes geschlossen werden - so sollen ein größtmögliches 
Monitoring und ein gründlicher Evaluierungsprozess der gemachten Fortschritte gesichert werden.

\section{Auswertung der Hypothesen und Process Tracing Analyse}

Vor dem soeben skizzierten politischen und historischen Hintergrund soll nun die Analyse der Hypothesen sowie ein Blick auf mögliche Mechanismen von Europäisierungsprozessen mittels Process Tracing erfolgen.

Hypothese I behandelte die Vorherrschaft von „Bottom-Up“-gegenüber „Top-Down“-Europäisierungsprozessen in "alten“ Mitgliedstaaten. Da die Hypothese primär die Situation in den pre-2004 EU-Mitgliedstaaten betrifft, soll hauptsächlich auf die Evaluation der Daten aus diesen Fallstudien (Südtirol und Bretagne) eingegangen werden.

Für die Fallstudie Südtirols bewahrheitet sich die Prävalenz von „Bottom-Up“-Prozessen nur teilweise, da die Entwicklung im Rahmen des momentan durchgeführten Autonomiekonvents auch eine „Top-Down“Dimension aufweist. Das Zweite Autonomiestatut von I973 muss an die Anforderungen des EU-Rechts sowie an moderne gesellschaftliche Herausforderungen angepasst werden; dies erfolgt im Rahmen eines Dialogs mit Parteien und der Zivilgesellschaft - dem sogenannten Autonomiekonvent. Es gibt einen misfit zwischen Normen des Autonomiestatuts und Vorgaben des EU-Rechts (ausgedrückt z.B. durch die EuGH-Urteile zur Gültigkeit der Sprachzertifikate sowie zur Verwendung des Deutschen vor Gericht - C 274/96; C 28I/98; C 322/13); dadurch entsteht Druck zur Anpassung (vgl. Börzel/ Risse 2003) - ein klassischer Europäisierungsprozess „Top-Down“. Dieser Prozess von „oben nach unten“ löst im Gegenzug einen „Bottom-Up“-Prozess im Rahmen eines zivilgesellschaftlichen Dialogs aus, bei dem die Überarbeitung des Autonomiestatuts unter Einbindung von möglichst vielen Stakeholdern erfolgen soll. Während es sich bei den Normen im Rahmen des EU-Rechts nicht um Bestimmungen zum Minderheitenschutz als solchem handelt, haben sie dennoch wichtige Auswirkungen auf die Situation der Minderheit (z.B. Vorgaben im Bereich Nicht-Diskriminierung). Die erste Phase des Konvents (Abhaltung sogenannter "open spaces", in den die BürgerInnen ihre Vorschläge einbringen konnte) wurde kürzlich beendet; das Gremium trifft sich jetzt, mit Beteiligung der gewählten zivilgesellschaftlichen VertreterInnen, in zweiwöchigen Abständen zur Ausarbeitung konkreter Maßnahmen. Der Fall des Autonomiekonvents verdeutlicht die Zweidimensionalität von Europäisierungsprozessen, die am selben Politikfeld ersichtlich sind und sich indirekt auch gegenseitig auslösen. Die Analyse des Südtiroler Fallbeispiels bringt also ein überraschendes Ergebnis zu Tage, welches laut
Bayesscher Logik, der Grundlage des Process Tracing, eine verstärkte Aussagekraft der gesammelten Beobachtungen vermuten lässt (Beach/Pedersen 2OI3). Im Rahmen der Analyse kann der Mechanismus dieses zweidimensionalen Europäisierungsprozesses nach der Klassifizierung von Beach und Pedersen (2013) als institutioneller Mechanismus angesehen werden; da die involvierten Akteure durch Institutionen und deren Normen in eine bestimmte Handlungsrichtung gelenkt werden. Der Mechanismus wird durch den misfit zwischen EU-Recht und Autonomiestatut in Gang gesetzt; die Akteure entschließen sich, diesen Konflikt zu beseitigen und das Statut abzuändern bzw. neu zu interpretieren. $\mathrm{Da}$ sie dies durch einen partizipativen Prozess (den Autonomiekonvent) beabsichtigen, findet als zweiter Teil des Mechanismus eine zivilgesellschaftliche „BottomUp“-Bewegung statt. Dies kann als struktureller Mechanismus interpretiert werden, da die Akteure von politischen Gegebenheiten zum Handeln gezwungen werden und dabei einer Rational Choice Logik folgen - sollen Konflikte und höhere Anpassungskosten vermieden werden, muss eine Adaptierung oder Neuinterpretation des Autonomiestatus erfolgen. Die Auswertung der parlamentarischen Fragen an die Europäische Kommission verdeutlicht, dass „Bottom-Up“-Prozesse im Südtiroler Fall meist als Versuch der Erreichung konkreter Ziele auftreten, und zwar in Bereichen, in denen die EU Handlungskompetenz hat; z.B. gibt es Anfragen zur Verfügbarkeit von deutschsprachigen Online-Inhalten, die durch geografische Restriktionen nur in Deutschland und Österreich erworben werden können. Hier weist die Kommission in ihrer Antwort auf eine bevorstehende Harmonisierung des Patentrechts in Europa hin.

Die Bretonen betreiben hingegen auch sehr starkes Lobbying für Minderheiten- und Sprachenschutz im Allgemeinen; da sie über keinen eigenen MEP verfügen, übernehmen meist Vertreter anderer Minderheiten diese Aufgabe. Da es um stark grundsatz- und werteorientierte Fragen geht, handelt es sich dabei um einen ideellen Mechanismus. Im Fall der Bretagne gibt es nur sehr geringe Möglichkeiten für „Top-Down“-Prozesse, da der Staat seine Gate-Keeper-Funktion weiterhin wahrnimmt, und der non-acquis-Transfer von Normen ausbleibt. Die EU kann die Minderheiten in Frankreich im Rahmen von Programmen zur Förderung der Sprache und Kultur unterstützen, ein offizielles Eingreifen ist ihr jedoch in Abwesenheit von Konditionalität als Werkzeug nicht möglich. Die Bretonen verfügen über eine sehr aktive Zivilgesellschaft, die sich laut DG Justiz auch immer wieder an die EU-Institutionen wendet. Es handelt sich dabei aber meist um allgemeine Forderungen zur Anerkennung als Minderheit und zu kollektiven Rechten, bei denen die EU keine Kompetenz hat; eine Intervention wäre nur im Einzelfall z.B. bei Diskriminierung aufgrund der ethnischen Zugehörigkeit möglich. 
Allgemein lässt sich ein deutlicher Anstieg der Thematisierung von Minderheiten-Issues auf EU-Ebene feststellen: In der Legislaturperiode 2009-20I4 gab es 26 parlamentarische Fragen, die sich mit Minderheiten befassten oder von ihren Vertretern gestellt wurden; in den Legislaturen 1999-2004 und 2004-2009 waren es zusammen nur 16 Fragen. Eine Verlagerung der Aktivitäten auf die europäische Ebene als „Bottom-Up“-Prozess ist also durchaus spürbar. Diese Entwicklung deckt sich mit den Aussagen der MinderheitenvertreterInnen, die die EU als wichtigen Rahmen für ihre Aktivitäten, als Forum für Zusammenarbeit mit anderen Minderheiten und als zusätzlichen Garant für Schutz vor staatlichen Repressalien sehen.

In Schlesien und Istrien treten ebenfalls „BottomUp“ Entwicklungen auf. Diese sind allerdings schwächer ausgeprägt und konzentrieren sich vor allem auf den kulturellen Sektor und Möglichkeiten der Zusammenarbeit im Rahmen der historischen, grenzüberschreitenden Regionen. Während die italienische Minderheit in Istrien auch durchaus politisch aktiv ist, ist die deutsche Minderheit in Schlesien diesbezüglich sehr vorsichtig; auch ein zu großes Engagement in der schlesischen Autonomiebewegung wird gescheut, da man Separatismusvorwürfe und das negativ geprägte Öffentlichkeitsbild der Minderheit fürchtet. Einen interessanten „Bottom-Up"-Prozess stellt die Zusammenarbeit der deutschen Minderheit, die über keinen eigenen Vertreter im EU-Parlament verfügt, mit EU-Parlamentariern anderer Minderheiten dar; da daraus aber bisher keine konkreten politischen Forderungen entstanden sind, handelt sich dabei um einen rein ideellen Mechanismus.

Hypothese 2 sprach von der Möglichkeit, auf europäischer Ebene Druck auf einen nicht kollaborierenden Nationalstaat auszuüben; von dieser Möglichkeit macht die deutschsprachige Minderheit in Südtirol allerdings wenig Gebrauch, da die Diskussionsbasis mit dem Mitgliedstaat offen ist und die meisten Angelegenheiten bereits auf nationaler Ebene geklärt werden können. Lobbying auf EU-Ebene wird dort betrieben, wo es konkrete Ansatzpunkte gibt, die innerhalb der EU-Kompetenzen liegen (z.B. Anfragen zur Verfügbarkeit von deutschsprachigem Online-Inhalt in Italien oder zum Erwerb von Decodern für den Empfang deutscher Programme); zudem wird die EU im Rahmen der Intergroup für Minderheiten des Europäischen Parlaments sowie von NGOs wie der FUEN als Ebene zur Vernetzung und Kollaboration mit anderen Minderheiten gesehen.

Die Bretonen hingegen nutzen die Möglichkeit, sich für ihre Anliegen auf europäischer Ebene Gehör zu verschaffen und dabei auch Druck auf den französischen Nationalstaat auszuüben. Die EU wird in mehreren Interviews als Zufluchtsstelle bezeichnet, wenn auf nationaler Ebene nichts erreicht werden kann. Auch wenn es hier keine Kompetenzen gibt, ist Europa trotzdem der primäre Ansprechpartner und auch Hoffnungsträger - es gibt also eine starke „Bottom-Up“-Dimension, die aber nur hinsichtlich Vernetzung mit anderen Minderheiten und kultureller Kollaboration Früchte trägt; eine direkte Einflussnahme ist aber nicht möglich. Dies wird auch aus den Interviews mit den VertreterInnen des DG Justiz deutlich, die viele Anfragen seitens der Bretonen erhalten, dabei meist aber nur auf die mangelnde Kompetenz der EU in generellen Minderheitenangelegenheiten verweisen können; ein Aktivwerden wäre nur in spezifischen, individuellen Diskriminierungsbeständen möglich.

Wie bereits bei der Analyse von Hypothese 2 angesprochen, verfügt die deutsche Minderheit in Schlesien, wie die Bretonen, über keinen eigenen Vertreter im EUParlament; man arbeitet aber eng mit Parlamentariern anderer Minderheiten zusammen. Es gibt eine gute Verhandlungsbasis zwischen Minderheit und Nationalstaat und wie im Fall Südtirols werden eher konkrete Themen an die EU-Ebene herangetragen; zudem erfolgen transnationale Kollaborationen im kulturellen Sektor.

Auch die italienische Minderheit in Istrien nutzt vorwiegend die Möglichkeit zum Austausch und zur Vernetzung im kulturellen Bereich, während konkrete politische Anliegen meist auf nationaler Ebene geregelt werden. Bisher wendet sich die italienische Minderheit, im Gegensatz zur serbischen, auch kaum direkt an die EU-Institutionen.

Hypothese 3 ging von „Top-Down“-Prozessen als vorherrschender Art von Europäisierung in den "neuen" Mitgliedstaaten aus, auf Grund der in der Forschung vorherrschenden Annahme einer eher schwachen $\mathrm{Zi}$ vilgesellschaft und der Möglichkeit zum Einsatz von Konditionalität im Beitrittsprozess. Für die „alten“ Mitgliedstaaten konnten, bis auf den bereits besprochenen überraschenden Prozess in Südtirol im Rahmen der Überarbeitung des Autonomiestatuts, keine „TopDown"-Prozesse festgestellt werden; in der Bretagne werden diese durch die Gate-Keeper-Funktion des Nationalstaats verhindert. Die Analyse von Hypothese 3 wird sich daher auf „Top-Down“-Prozesse in den neuen Mitgliedstaaten konzentrieren.

Generell stimmt die Annahme, dass „Top-Down"Bewegungen bisher die vorherrschende Art von Europäisierungsprozessen in den untersuchten post-2004 Mitgliedstaaten darstellen; der EU-Einfluss in der Beitrittsphase wird als sehr wichtig für Fortschritte im minderheitenpolitischen Sektor angesehen. Kritischer zu betrachten ist die postulierte Schwäche der Zivilgesellschaft, welche sich in der Analyse nicht bewahrheitet hat. Die deutsche Minderheit in Schlesien spricht zwar selbst von einer vermeintlich schwachen Zivilgesellschaft, ist aber sehr aktiv, vor allem im kulturellen Bereich. Politisch tritt die Minderheit eher vorsichtig auf, was dem in der Vergangenheit sehr negativ geprägten 
Bild in der Öffentlichkeit zuzuschreiben sein dürfte; man versucht daher, keinen eigenen politischen „Arm“ zu entwickeln, sondern Kollaborationen mit polnischen Parteien aufzubauen, bisher allerdings mit wenig Erfolg. Gerade in der europäischen Zusammenarbeit ist die deutsche Minderheit allerdings sehr involviert, was z.B. auch das Engagement in der FUEN als eine der Initiatoren der abgelehnten Bürgerinitiative zum Minderheitenschutz zeigt. Die zivilgesellschaftlichen Strukturen mussten nach der Repression im Kommunismus erst wieder aufgebaut werden, sind aber nun vor allem auch durch die europäische Ausrichtung und die Arbeit im kulturellen Bereich wieder zu einem wichtigen Faktor geworden.

Der Fall der italienischen Minderheit in Kroatien ist ähnlich gelagert; auch hier kann von einer schwachen Zivilgesellschaft keine Rede sein. Während die zivilgesellschaftliche Arbeit in Polen unter dem Kommunismus nur schwer fortgeführt werden konnte, verfügte Istrien auch in der jugoslawischen Zeit bereits über eine gut ausgeprägte Repräsentations- und Vernetzungsstruktur sowie einen relativ weitreichenden Minderheitenschutz. Die italienische Minderheit ist sowohl auf dem kulturellen als auch auf dem politischen Sektor sehr aktiv, was mit der Kontinuität der Tätigkeit unter dem Kommunismus und dem geringeren Sprachverlust zusammenhängen dürfte. Auch das Öffentlichkeitsbild der Minderheit ist nicht negativ geprägt, was ein starkes Auftreten als Interessensvertretung auf nationaler und europäischer Ebene erleichtert. Die Möglichkeiten zur Vernetzung und Kollaboration, die auch durch den EU-Beitritt geschaffen wurden, werden von den Angehörigen der Minderheit als positiv gewertet und stark in Anspruch genommen (z.B. Zusammenarbeit in der Euregio „Ohne Grenzen“, eigenes Büro der Region in Brüssel). Während Konditionalität im kroatischen Beitrittsprozess durchaus eine Rolle spielte, vor allem im Fall der serbischen Minderheit sowie der Zusammenarbeit bei der Verfolgung von Kriegsverbrechen, war die italienische Minderheit davon kaum betroffen - der Großteil des Minderheitenschutzes war bereits implementiert worden.

Für beide Fallstudien ist ein institutioneller „TopDown"-Prozess im Beitrittsprozess erkennbar, wobei dieser im Fall Schlesien als wichtiger anzusehen ist als im Fall der italienischen Minderheit in Istrien, die bereits vorher sehr gut geschützt war. In Polen gab es bereits vorher Schutzmaßnahmen, jedoch bot das Umfeld des EU-Beitritts laut Minderheitenvertretern den nötigen Anstoß zur letztendlichen Beschlussfassung und Durchführung der Minderheitengesetzgebung. In Kroatien wurde laut EU-BeamtInnen erstmals eine neue Methode im Beitrittsprozess ausprobiert, die für mehr Nachhaltigkeit sorgen soll: Das Kapitel Menschenrechte wurde als erstes eröffnet und als letztes geschlossen, um mehr Monitoring in der Verhandlungsphase gewährleisten zu können. Dieser Ansatz beinhaltet auch eine verstärkte Kollaboration mit Minderheitenvertretern; für die italienische Minderheit trifft dies allerdings nicht zu, da die EU-Kommission hier keine Kritikpunkte angemerkt hatte. Beide Länder werden nun in die Rolle eines „alten“ Staates fallen, in dem die EU keinen politischen Einfluss mehr auf die Minderheitenpolitik ausüben kann; zukünftige Europäisierungsprozesse werden also verstärkt „Bottom-Up“ ablaufen müssen. Hier sind vor allem Kollaborationen auf EU-Ebene und der Austausch von Best Practices zu nennen, die dann auf die subnationale Ebene „importiert“ werden können (z.B. durch Zusammenarbeit mit anderen Minderheiten bei der Erstellung von Schulbüchern; hier gab es eine Kollaboration zwischen Schlesien und Südtirol). Auch kulturelle Programme wie z.B. Grundtvig werden von den Minderheiten zur internationalen Vernetzung genutzt. Eine weitere wichtige Rolle für „Bottom-Up“-Entwicklungen spielt die Zusammenarbeit in den Euregio-Verbünden; hier werden Kollaborationen im Rahmen der historischen Regionen gefördert, die durch Grenzen getrennt wurden, aber ähnliche Ziele und Anforderungen aufweisen und verstärkt zusammenarbeiten möchten. Istrien ist bereits in der Euregio „Ohne Grenzen" aktiv, und die deutsche Minderheit strebt eine schlesische Euregio-Kollaboration an (zusammen mit Deutschland und Tschechien).

Wie in Hypothese 4 formuliert spielen nationale Faktoren eine wichtige Rolle, da die Kompetenz hinsichtlich Minderheitenpolitik weiterhin in den Händen der Nationalstaaten liegt. Sie können Europäisierungsprozesse fördern oder verlangsamen (also Katalysatorbzw. Gate-Keeper-Funktion ausüben), sie aber nicht gänzlich stoppen. Am Südtiroler Beispiel sind vor allem die Einstellung des Staates zu Minderheiten und die internationale Verankerung der Autonomie wichtig; die Republik Italien hat Minderheitenschutz sowohl in der Verfassung als auch in der Gesetzgebung festgeschrieben (Art. 6 der Verfassung, Gesetz von 1999) und die Minderheit verfügt über eine stabile parlamentarische Vertretung in Rom - eine Präsenz starker Veto-Player ist nicht gegeben, auch wenn die Sparmaßnahmen und Zentralisierungen im Rahmen der Finanzkrise auch die Übertragung von Kompetenzen und Ressourcen auf die Autonomieebene behindern.

Im Fall Bretagne spielt der Staat eine sehr wichtige Rolle und schränkt den Einfluss von Europäisierungsprozessen durch sein striktes Gleichheitsverständnis durchaus ein. Da keine Anerkennung von Minderheiten- und Gruppenrechten möglich ist, sind viele der Ziele der Bretonen von vornherein blockiert oder aber noch in weiter Ferne. Ein Beispiel dafür ist die geplante Ratifikation der Charta für Regional- und Minderheitensprachen, die nach Zustimmung der Nationalversamm- 
lung durch den Senat wieder blockiert wurde. Konkrete Schritte hin zu einer Ratifizierung sind derzeit nicht zu erwarten; ein deutlicher Kurswechsel ist auch nach den Präsidentschaftswahlen 2017 unwahrscheinlich.

Bei der deutschen Minderheit in Schlesien ist es vor allem das negative Image, welches ein größeres politisches Engagement verhindert (auch z.B. im Rahmen der Autonomiebewegung); hier spielt also nicht der Staat, sondern das Öffentlichkeitsbild eine beschneidende Rolle und fordert eine gezielte Zurücknahme eigener politischer Aktivitäten, um vorherrschende Stereotype nicht weiter zu befeuern. In Istrien hingegen war es ein Regierungswechsel im Jahr 2000, der den Weg in Richtung Europa endgültig öffnete und auch die Ausrichtung des Landes hinsichtlich Minderheiten veränderte. Eine solche Umorientierung wäre unter Tudjman nicht möglich gewesen; politische Kräfte und Machtwechsel spielten also eine zentrale Rolle. Nationale Veto-Player konnten durch den sehr starken Pull-Faktor eines EUBeitritts überwunden werden.

\section{Schlussfolgerungen}

In einer finalen Schlussbetrachtung sollen nun die im Rahmen des Testens der Hypothesen sowie der ProcessTracing-Analyse gesammelten Erkenntnisse nochmals in ein vergleichendes Bild gesetzt werden. Als eines der Hauptergebnisse kann gelten, dass die Unterscheidung zwischen "alten“ und "neuen“ EU-Mitgliedstaaten auf einer längeren Zeitebene nicht aufrecht erhalten werden kann: Auch wenn die EU bei den 2004 und später beigetretenen Mitgliedern Konditionalität als Werkzeug zur Verfügung hatte, übt dies langfristig und teilweise schon nach der Beitrittsphase keinen Einfluss mehr aus. Die „neuen“ Staaten werden also, trotz genauerer Evaluierung ihrer Minderheitenpolitik im Beitrittsprozess, im Laufe der Zeit zu „alten“ Staaten, in denen das Minderheitenthema wie in der restlichen EU rein innerstaatlich behandelt wird und fest in der Kompetenz der einzelnen Mitgliedstaaten liegt. Einfluss könnte noch durch im Beitrittsprozess gemachte Zugeständnisse (vgl. Konzept des „rhetorical entrapment“, Schimmelfennig 2008) ausgeübt werden; da Minderheitenschutz nicht Teil des acquis communautaire ist, kann die $\mathrm{EU}$ hier nicht direkt eingreifen.

Die Daten aus den Fallstudien belegen die Annäherung zwischen "alten" und "neuen“ Mitgliedstaaten nach dem Beitritt; nachdem Einflussnahme durch Konditionalität nicht mehr möglich ist, orientieren sich die Minderheiten um, und „Bottom-Up“-Prozesse lösen „TopDown"-Entwicklungen als häufigste Art von Europäisierung ab. Diese Entwicklung ist schon kurz nach dem Beitritt spürbar. Auch in Kroatien, welches erst seit 2013 zur EU gehört, sind die Minderheiten bereits zivilgesell- schaftlich sehr vernetzt und haben viele ihrer Aktivitäten auf die EU-Ebene verlagert oder diese zumindest als politisches Forum dazugewonnen. In Polen wurde auf die wichtige Wirkung von EU-Konditionalität als Katalysator für Veränderungen während des Beitrittsprozesses hingewiesen; auch hier zeigt sich aber eine deutliche Verlangsamung der Entwicklungen seit 2004 und eine größere Rolle der Zivilgesellschaft der deutschen Minderheit bei der Verfolgung ihrer Ziele auch auf europäischer Ebene. Im Gegensatz zur italienischen Minderheit in Istrien ist die deutsche Minderheit in Schlesien aber vor allem kulturell gut vernetzt und aktiv; größere politische Einbindung wird hauptsächlich vom weiterhin teilweise negativen Image der Minderheit verhindert. Die prognostizierte Schwäche der Zivilgesellschaft in „neuen“ Mitgliedstaaten kann für die untersuchten Fallstudien nicht festgestellt werden; sowohl in Istrien als auch in Schlesien sind die zivilgesellschaftlichen Akteure sehr aktiv - bedingt durch die jeweilige Vergangenheit der Minderheit im politischen und kulturellen oder nur im kulturellen Sektor. Die Zivilgesellschaft in beiden Ländern ist international gut vernetzt und schätzt die Möglichkeiten zur Zusammenarbeit, die die EU bietet; nach dem EU-Beitritt und dem Übergang vom "neuen“ zum „alten“ Mitgliedstaat verstärkt sich ihre Relevanz weiter.

Im Fall der Minderheiten in „alten“ EU-Staaten hat sich die angenommene Prävalenz von „Bottom-Up“-Prozessen bewahrheitet; sehr überraschend ist hingegen, dass durchaus auch „Top-Down“-Prozesse zu beobachten sind, wie z.B. die Adaptierung bzw. Neu-Interpretierung des Autonomiestatus in Südtirol. Auch wenn es bei solchen „Top-Down“-Prozessen nicht direkt um Minderheitenschutzmechanismen geht, so haben sie doch großen Einfluss auf die tägliche Lebensrealität der Minderheiten, wie die oben erwähnten Entscheidungen des EuGH zur Gleichbehandlung von EU-Bürgern. Der Nationalstaat bleibt allerdings weiterhin der primäre Ansprechpartner für Minderheitenanliegen, da hier die Kompetenzen liegen; „Upload“-Prozesse nach Europa werden vor allem zu konkreten Themen, bei denen die EU auch einen Handlungsspielraum hat, sichtbar (z.B. grenzüberschreitende Verfügbarkeit von Online-Inhalten). Anders gelagert ist der Fall, wenn der Mitgliedstaat nicht auf die Anliegen der Minderheit eingeht oder deren Existenz sogar grundsätzlich abstreitet; die Bretonen nutzen daher die europäische Ebene auch für Lobbying für generelle Schutzmaßnahmen - die EU kann hier allerdings nur erklären, nicht zuständig zu sein. Appelle an "Europa“ bieten aber die Möglichkeit, auf das Thema aufmerksam zu machen und ein Problembewusstsein zu schaffen. Gerade im Umfeld des Europäischen Parlaments besteht eine gute Kollaborationsbasis, so werden z.B. Minderheiten ohne MEP von anderen Minderheitenvertretern durch parlamentarische Fragen 
repräsentiert. Diese Entwicklung hin zu europäischen Kollaborationen könnte auch dem Ruf des Parlaments entgegenwirken, nur eine Stellvertreterfunktion für nationale politische Prozesse einzunehmen und möglicherweise einen Legitimitätsgewinn bedeuten.

Bei den untersuchten Mechanismen lässt sich eine Vorherrschaft von institutionellen Mechanismen in „Top-Down“-Prozessen und sowohl institutionellen als auch ideellen Mechanismen in „Bottom-Up“-Prozessen feststellen. Faktoren auf nationaler Ebene, vor allem die Möglichkeit und Qualität der Zusammenarbeit mit dem Mitgliedstaat, bleiben weiterhin sehr wichtig - gibt es eine gute Kommunikation zwischen Staat und Minderheit, können viele Themen bereits auf nationaler Ebene gelöst werden und ein „Upload“ Richtung Europa ist nicht mehr notwendig. Die Präferenzen des Nationalstaates hinsichtlich des Minderheitenschutzes äußern sich bereits in der Verfassung bzw. Gesetzgebung, Regierungswechsel oder Veto-Player können aber ebenfalls noch eine wichtige Rolle spielen.

Der Charakter von Europäisierung als zweidimensionaler Prozess, der sowohl „Bottom-Up“- als auch „TopDown"-Komponenten umfassen kann, wird am Beispiel Südtirols und dem Autonomiekonvent nochmals besonders deutlich. Hier löst ein „Top-Down“-Prozess, der misfit zwischen EU-Recht und Autonomiestatut und der daraus resultierende Anpassungsdruck, einen „BottomUp“-Prozess aus, nämlich den Autonomiekonvent, bei dem die Adaptierung oder Neuinterpretation des Statuts unter Einbeziehung möglichst vieler relevanter Stakeholder passieren soll. Ein politischer Prozess oder eine Entwicklung kann also sowohl „Top-Down“- als auch „Bottom-Up“-Komponenten beinhalten. Europäisierung als zweidimensionaler Vorgang könnte daher ein nützliches Analyse-Framework sein, wenn man Prozesse von zwei Perspektiven beobachten möchte, z.B. für gemeinsame Aktivitäten transnationaler Netzwerke als „Bottom-Up“-Prozess und vorhergehendes oder nachfolgendes Policy-Making auf EU-Ebene als „Top-Down“Komponente. Auch die Einbindung von zivilgesellschaftlichen Strukturen in den Politikprozess könnte im Rahmen zweidimensionaler Europäisierungsprozesse analysiert werden; die Entwicklung hin zu einer gesamteuropäischen Zivilgesellschaft bietet zudem einen interessanten zukünftigen Forschungsansatz.

\section{Literatur}

Anderini, Sabina (2006). L'Istria e la minoranza italiana nella questione jugoslava, 1974-1994, Rom.

ASTAT (2013). Statistisches Jahrbuch 20I3, http://www. provinz.bz.it/astat/download/Jahrbuch_20I3.pdf (Zugriff: 09.05.16).
Beach, Derek/Rasmus Brun Pedersen (2013). Processtracing methods. Foundations and guidelines, Ann Arbor.

Börzel, Tanja/Thomas Risse (2003). Conceptualising The Domestic Impact of Europe, in: Featherstone, Kevin/ Claudio M. Radaelli (Hg.): The politics of Europeanization, Oxford, 57-80.

Central Statistical Office of Poland (20I4). Population, http:// stat.gov.pl/en/topics/population/ (Zugriff: 09.05.16).

Cordell, Karl/Stefan Wolff(2005). Ethnic Germans in Poland and the Czech Republic: a comparative evaluation, in: Nationalities Papers, Vol. 33 (2), 255-276.

Croatian Bureau of Statistics (2OI3). Census 20II, http:// www.dzs.hr/default_e.htm (Zugriff: 09.05.16).

Cowles, Maria Green/James Caporaso/Thomas Risse (200I). Transforming Europe. Europeanization and domestic change, Ithaca.

Crepaz, Katharina (2016). The Impact of Europeanization on Minority Communities, Wiesbaden.

Della Porta, Donatella/Manuela Caiani (2009). Social movements and Europeanization, Oxford.

EuGH (1996). C 274/96 - Horst Otto Bickel \& Ulrich Franz, http://tinyurl.com/obquw56 (Zugriff: 09.05.I6).

EuGH (1998). C 28I/98 - Roman Angonese v Cassa di Risparmio di Bolzano SpA, http://curia.europa.eu/juris/ document $/$ document.jsf?text $=\&$ docid $=45323 \&$ pageI ndex $=$ o\&doclang $=E N \&$ mode $=1$ st $\&$ dir $=\& o c c=$ first $\& p$ art $=$ I\&cid $=93334$ (Zugriff: 09.05.I6).

EuGH (2OI3). C 322/13 - Ulrike Elfriede Grauel Rüffer v Katerina Pokorná, http://curia.europa.eu/juris/document/document.jsf?text $=\&$ docid $=I 49923 \&$ pageInde $\mathrm{x}=\mathrm{O} \&$ doclang $=$ en\& mode $=1 \mathrm{st} \&$ dir $=\&$ occ $=$ first $\&$ part $=$ I\& cid=93375 (Zugriff: 09.05.I6).

FUEN. Europäische Minderheiten, https://www.fuen. org/de/europaeische-minderheiten/allgemein/ (Zugriff: 09.05.16).

Graziano, Paolo/Peter Maarten Vink (2007). Europeanization. New research agendas, Houndsmills.

Keck, Margaret E../Kathryn Sikkink (1998). Activists beyond borders. Advocacy networks in international politics, Ithaca.

McCauley, Darren (20II). Bottom-Up Europeanization Exposed: Social Movement Theory and Non-state Actors in France, in: Journal of Common Market Studies, Vol, 49(5),IOI9-IO42.

Office Publique de la Langue Bretonne (2013). Situation Sociolinguistique, http://www.fr.opab-oplb.org/6-situation-sociolinguistique.htm (Stand: 09.05.16).

Pallaver, Günther (2OI4). South Tyrol's changing political system: from dissociative on the road to associative conflict resolution, in: Nationalities Papers, Vol. 42(3), 376-398.

Radaelli, Claudio M./Theofanis Exadaktylos (2010). New Directions in Europeanisation Research, in: Egan, 
Michelle P./Neill Nugent/William E. Paterson (Hg.): Research agendas in EU studies. Stalking the elephant. Basingstoke, 188-215.

Republik Italien (1999). Legge 482/99 Norme in materia di tutela delle minoranze linguistiche storiche, http:// www.camera.it/parlam/leggi/994821.htm (Zugriff: 09.05.16).

Republik Frankreich (1958). Verfassung der Republik Frankreich, http://www.assemblee-nationale.fr/ deutsch/8cb.asp (Zugriff: 09.05.16).

Republik Italien (1947). Verfassung der Republik Italien, http://www.landtag-bz.org/download/Verfassung _ Italien.pdf (Zugriff:09.05.16).

Schimmelfennig, Frank (2008). EU political accession conditionality after the 2004 enlargement: consistency and effectiveness, in: Journal of European Public Policy, Vol. 15(6), 918-937.

Schimmelfennig, Frank/Ulrich Sedelmeier (2004). Governance by conditionality: EU rule transfer to the candidate countries of Central and Eastern Europe, in: Journal of European Public Policy Vol. II (4),66I-679.

\section{Autorin}

Katharina Crepaz, geb. 1986, ist wissenschaftliche Mitarbeiterin (Post-Doc) am Max Planck Institut für Sozialrecht und Sozialpolitik (Fellow-Gruppe: „Dis[cover] ability and Indicators for Inclusion (2015-2020)“) sowie am Lehrstuhl Diversitätssoziologie der TU München. Studium der Politikwissenschaft, Anglistik und Amerikanistik sowie Skandinavistik an den Universitäten Innsbruck und Wien, Promotion (Ph.D.) in Politikwissenschaft an der Universität Innsbruck 2015 (Dissertation: The Impact of Europeanization on Minority Communities, Springer VS, 2016). Forschungsinteressen: Nationale und migrantische Minderheiten, Europäisierung, Zivilgesellschaft, politische Partizipation gesellschaftlich marginalisierter Gruppen, Inklusion, Separatismusund Regionalismusforschung. 
